\title{
Atributos físicos do solo sob diferentes preparos e coberturas influenciados pela distribuição de poros
}

Eurâimi de Q. Cunha ${ }^{1}$, Luís F. Stone ${ }^{2}$, José A. A. Moreira2 ${ }^{2}$ Enderson P. de B. Ferreira ${ }^{2} \&$ Agostinho D. D idonet ${ }^{2}$

RESUMO

Com este trabalho se propõe avaliar a influência da distribuição de poros sobre alguns atributos físicos do solo sob semeadura direta (SD) e preparo convencional (PC), cultivado com diferentes culturas de cobertura, no sistema de produção orgânica de feijão e milho. 0 trabalho foi conduzido em Santo Antônio de Goiás, GO, em Latossolo Vermelho distrófico. Em novembro de 2003 foram instalados quatro experimentos, dois em SD e dois em PC, um em cada manejo com feijão e o outro com milho. Foram comparados em blocos ao acaso com quatro repetições, crotalária, guandu, mucuna-preta, sorgo e pousio. Amostragens de solo das parcelas e de uma mata próxima foram realizadas nas profundidades de 0-0,10 e 0,10-0,20 m, em novembro de 2007, para determinação do teor de matéria orgânica (M.O.) e de atributos físicos do solo. 0 uso do solo sob vegetação de cerrado para a produção agrícola, independentemente do sistema de cultivo, resultou em redução na porosidade total (Pt), macroporosidade ( $\mathrm{M} \mathrm{p}$ ) e capacidade de aeração do solo (CAS). Os atributos físicos do solo foram afetados favoravelmente pela M.O. As variações em Pt, Mp, CAS e capacidade de água disponível do solo podem ser explicadas pela variação na distribuição do tamanho de poros do solo, principalmente daqueles com $\varnothing>0,075 \mathrm{~mm}$.

Palavraschave: Phaseolus vulgaris L., Zea mays, semeadura direta, porosidade do solo, capacidade de aeração do solo

\section{Soil physical attributes under different tillage systems and cover crops, as influenced by pore distribution}

\begin{abstract}
This study aimed to evaluate the influence of pore distribution on some physical attributes of soil under no-tillage (NT) and conventional tillage (CT) systems, cultivated with different cover crops, in organic production of common bean and corn. The work was carried out in Santo Antônio de Goiás, GO, on an Oxisol. In November 2003 four experiments were installed, two of them under NT and the other two under CT. In each soil tillage system, an experiment was conducted with corn and another with common bean. Sunn hemp, pigeon pea, velvet bean, sorghum, and fallow were compared in a randomized block design, with four replications. Samples were taken from soil layers of 0-0.10 and 0.10-0.20 m in N ovember 2007, in the plots and in a near by native forest (Cerrado), for determination of soil organic matter $(\mathrm{OM})$ content and physical attributes. Cerrado soil use for agricultural production, regardless of tillage system, resulted in decrease of soil total porosity (TP), macroporosity $(\mathrm{Mp})$, and soil air capacity $(\mathrm{SAC})$. Soil physical attributes were favorably affected by $0 \mathrm{M}$. Variations in TP, M p, SAC, and available soil water capacity can be explained by variation in soil pore size distribution, mainly by those with $\varnothing>0.075$ $\mathrm{mm}$.
\end{abstract}

Key words: Phaseolus vulgaris L., Zea mays, no-tillage, soil porosity, soil air capacity

\footnotetext{
1 Doutorando do Curso de Pós-Graduação em Agronomia da UFG, Goiânia, GO. Fone (62) 3534-9801. E-mail:euraimi@yahoo.com.br ${ }^{2}$ Embrapa Arroz e Feijão, CP 179, CEP.75375-000, Santo Antônio de Goiás, G0. Fone (62) 3533-2151. E-mail: stone@cnpaf.embrapa.br; jaloisio@cnpms.embrapa.br; enderson@cnpaf.embrapa.br; didonet@cnpaf.embrapa.br
} 


\section{INTRODUÇÃO}

A porosidade do solo é constituída por poros de diferentes tamanhos, formas e continuidade, que influenciam a infiltração, o armazenamento e a drenagem da água; o movimento e a distribuição dos gases e o crescimento das raízes (Kay \& Vandenbygaart, 2002).

A distribuição dos poros na matriz do solo desempenha papel fundamental nas relações entre as fases sólida, líquida e gasosa, determinando a evolução espacial e temporal dos processos que envolvem o movimento de água no solo. Assim sendo, a distribuição dos poros por seus tamanhos condiciona o comportamento físico-hídrico do solo, influenciando a potencialidade agrícola dos solos.

Para conhecimento mais aprofundado da permeabilidade do solo, dos processos de infiltração e redistribuição, da capacidade de retenção, e da extensão na qual a zona radicular é suprida com ar, a diferenciação da porosidade total, de acordo com o tamanho dos poros do solo, é essencial (Ribeiro et al., 2007).

Os sistemas de preparo do solo e de culturas têm influência significativa na estrutura do solo e nos fluxos de água e ar (Argenton et al., 2005). O não revolvimento do solo na semeadura direta induz à compactação e à diminuição do volume de macroporos da camada superficial (Oliveira et al., 2002). Apesar disso, alguns trabalhos destacam um balanço positivo entre porosidade de aeração e armazenamento de água no solo, sob este sistema de manejo (Stone \& Silveira, 1999). Oliveira et al. (2002) verificaram, em comparação com o Cerrado nativo, que o solo cultivado, tanto na semeadura direta como com preparo com arado de discos, sofreu redução na macroporosidade e aumento na porosidade de retenção de água. Na profundidade de 0 a $5 \mathrm{~cm}$ a semeadura direta foi o sistema que mais alterou a estrutura do solo, apresentando menor volume de poros com diâmetros superiores a 0,145 $\mathrm{mm}$; entretanto, apresentou maior volume de poros entre 0,009 e $0,050 \mathrm{~mm}$ de diâmetro em relação ao preparo com arado de discos, o que é benéfico por significar água mais disponível às plantas. Spera et al. (2000) afirmaram que o teor de água disponível está relacionado ao volume de poros entre 0,003 e $0,030 \mathrm{~mm}$ de diâmetro e não ao de poros com diâmetro menor que $0,003 \mathrm{~mm}$.

Ribeiro et al. (2007), comparando seis solos da região de Lavras, MG, observaram que os poros com diâmetro entre 0,009 e $0,0375 \mathrm{~mm}$ exerceram pouca influência sobre a densidade e porosidade total do solo, índice de vazios e condutividade hidráulica saturada.

Algumas plantas podem minimizar os efeitos negativos da degradação dos solos, por meio da melhoria na agregação e estrutura devido, principalmente, à ação mecânica das raízes ou pela excreção de substâncias com ação cimentante e, indiretamente, pelo fornecimento de nutrientes à fauna do solo, através da adição de restos culturais. A melhor agregação do solo reflete na densidade e porosidade do solo e na retenção de água (Sousa Neto et al., 2008).
A avaliação da distribuição do tamanho ou classe de poros tem permitido identificar diferenças entre sistemas de manejo do solo ou de culturas, com relação às condições físicas do solo. Assim, este trabalho objetivou avaliar as alterações na distribuição dos poros do solo cultivado com diferentes culturas de cobertura, em semeadura direta e preparo convencional do solo, no sistema de produção orgânica de feijão e milho, procurando verificar sua influência sobre alguns atributos físicos do solo.

\section{MATERIAL E MÉTODOS}

O trabalho foi conduzido na Fazenda Capivara, da Embrapa Arroz e Feijão, localizada no município de Santo Antônio de Goiás, GO, cujas coordenadas geográficas são: latitude $16^{\circ} 28^{\prime} 00^{\prime \prime} \mathrm{S}$, longitude $49^{\circ} 17^{\prime} 00^{\prime \prime} \mathrm{W}$, e altitude de $823 \mathrm{~m}$. O clima, conforme classificação de Köppen, é Aw, tropical de savana, megatérmico. O regime pluvial é bem definido, com período chuvoso de outubro a abril e seco de maio a setembro, com precipitação média anual de $1460 \mathrm{~mm}$ (Silva et al., 2002). O solo do local é um Latossolo Vermelho distrófico, de textura franco argilosa, com $410 \mathrm{~g} \mathrm{~kg}^{-1}$ de areia, $270 \mathrm{~g} \mathrm{~kg}^{-1}$ de silte e $320 \mathrm{~g} \mathrm{~kg}^{-1}$ de argila, na camada de 0,00-0,20 m. Antes da implantação dos experimentos foram aplicados em toda a área e incorporados com grade aradora, $1620 \mathrm{~kg} \mathrm{ha}^{-1}$ de fosfato natural Arad ( $33 \%$ de $\mathrm{P}_{2} \mathrm{O}_{5}$ ) e $2000 \mathrm{~kg} \mathrm{ha}^{-1}$ de calcário. A vegetação original da área experimental era do tipo Cerradão e vinha sendo cultivada no sistema convencional de preparo do solo (gradagens aradora e niveladora) com a rotação milho e soja.

Em novembro de 2003 foram instalados quatro experimentos que vêm sendo conduzidos segundo os preceitos da produção orgânica. Dois experimentos foram conduzidos em semeadura direta e, nos outros dois, foi feito o preparo convencional do solo com grades aradora e niveladora. Em cada sistema de manejo do solo um experimento foi conduzido com a cultura do milho e outro com a do feijão das águas. Em todos os experimentos foram comparadas, no delineamento de blocos ao acaso, com quatro repetições, as culturas de cobertura: crotalária (Crotalaria juncea), guandu (Cajanus cajan (L.) Millsp), mucuna-preta (Mucuna aterrima), sorgo vassoura (Sorgum technicum) e pousio (vegetação espontânea). A vegetação espontânea era constituída, basicamente, de picão preto (Bidens pilosa), capim pé-de-galinha (Eleusine indica) e leiteiro (Euphorbia heterophylla), com menores ocorrências de braquiária (Brachiaria decumbens), corda de viola (Ipomoea grandifolia), caruru (Amaranthus deflexus), erva de santa luzia (Chamaisice viridis L.), trapoeraba (Commelina benghalensis), mentrasto (Ageratum conyzoides), beldroega (Portulaca oleracea), guanxuma (Sida cordifolia) e maria pretinha (Solanum americanum Mill).

As culturas de cobertura foram semeadas em abril e conduzidas no sistema de semeadura direta. Por ocasião da semeadura do milho e feijão, em novembro, foram ma- 
nejadas com rolo-faca e deixadas sobre o solo (semeadura direta) ou incorporadas (preparo convencional). Elas foram semeadas no espaçamento de 0,45 m entre linhas, utilizando-se 60 sementes por metro de crotalária, guandu e sorgo e 20 sementes por metro de mucuna. O feijoeiro (Phaseolus vulgaris L.), cv. Pérola, foi semeado no mesmo espaçamento, com 16 sementes por metro e o milho (Zea mays L.), cv. AG 1051, no espaçamento de 0,90 m, com 5 sementes por metro. A área das parcelas com feijão era de $27,00 \mathrm{~m}^{2}(2,70 \times 10,00 \mathrm{~m})$ e as com milho de $54 \mathrm{~m}^{2}(5,40$ $\mathrm{x} 10,00 \mathrm{~m})$.

Em novembro de 2007 realizou-se amostragem de terra para análise físico-hídrica nas profundidades de 0,00-0,10 e $0,10-0,20 \mathrm{~m}$ e se retiraram amostras de uma mata próxima aos experimentos (Cerradão), representativa da vegetação original. Quatro amostras foram coletadas por profundidade estudada, sendo uma deformada e três não deformadas. As amostras com estrutura deformada foram utilizadas para determinar a textura do solo pelo método da pipeta, a densidade de partículas (Dp) pelo método do balão volumétrico e o teor de matéria orgânica pelo método de Walkley \& Black (EMBRAPA, 1997). Duas amostras não deformadas, coletadas em cilindros de $5 \mathrm{~cm}$ de diâmetro e $5 \mathrm{~cm}$ de altura, foram usadas para determinação da densidade do solo (Ds) e microporosidade. Calculou-se a porosidade total $(\mathrm{Pt})$ pela equação: $\mathrm{Pt}=(1-\mathrm{Ds} /$ $\mathrm{Dp})$ e a macroporosidade (Mp) foi obtida pela diferença entre a porosidade total e a microporosidade (EMBRAPA, 1997). A outra amostra não deformada foi usada para determinação das curvas de retenção de água pelo método da centrífuga (Freitas Júnior \& Silva, 1984). As curvas características de água no solo foram ajustadas por meio de regressão não-linear utilizando-se o modelo matemático proposto por Genuchten (1980).

Calculou-se a capacidade de aeração do solo (CAS) segundo a relação (Reynolds et al., 2002):

$$
\mathrm{CAS}=(\mathrm{Pt}-\mathrm{CC}) / \mathrm{Pt}
$$

em que CC é a capacidade de campo, considerada igual ao conteúdo de água no solo a tensão de $8 \mathrm{kPa}$, expressa $\mathrm{em} \mathrm{m} \mathrm{m}^{3} \mathrm{~m}^{-3}$.

A capacidade de água disponível (CAD) foi calculada pela diferença entre o conteúdo de água a tensão de 8 $\mathrm{kPa}$ e a tensão de $1500 \mathrm{kPa}$ (ponto de murcha), multiplicada pela espessura da camada considerada.

Realizou-se o cálculo do diâmetro dos poros para as tensões de 2, 4, 6, 8, 10, 33 e $1500 \mathrm{kPa}$, por meio da fórmula que estabelece a relação entre ele e a tensão da água retida em tubos capilares:

$$
\varnothing=(4 \sigma \cos \varphi)(g \rho h)^{-1}
$$

em que $\varnothing=$ diâmetro dos poros, em mm; $\sigma$ - tensão superficial da água, em dina $\mathrm{cm}^{-1} ; \varphi$ - ângulo de contato entre a água e a parede do capilar; g - aceleração da gravidade, em $\mathrm{cm} \mathrm{s} \mathrm{s}^{-2} ; \rho$ - densidade da água, em $\mathrm{g} \mathrm{cm}^{-3} \mathrm{e}$ h - altura da coluna de água, equivalente à tensão aplicada, em kPa.

A porcentagem de poros com diâmetro superior ao calculado para cada tensão (V) foi obtida pela expressão:

$$
\mathrm{V}=100[1-(\mathrm{Pt}-\theta) / \mathrm{Pt}]
$$

em que $\mathrm{Pt}=$ porosidade total do solo, em $\mathrm{m}^{3} \mathrm{~m}^{-3}$ e $\theta-$ umidade volumétrica, correspondente à tensão utilizada para o cálculo do diâmetro dos poros, em $\mathrm{m}^{3} \mathrm{~m}^{-3}$.

Com os dados obtidos foram construídas curvas de distribuição de poros, isto é, $\varnothing \mathrm{x} \mathrm{V}$, para todos os tratamentos.

Os dados foram submetidos a análise de variância por profundidade, utilizando-se o procedimento GLM do programa estatístico SAS (SAS Institute, 1999), sendo as médias comparadas pelo teste de Tukey a 5\% de probabilidade. As médias dos tratamentos foram comparadas com as obtidas no solo da mata nativa, pelo teste de Dunnett a 5\%. Realizaram-se análises de regressão linear entre as variáveis estudadas considerando-se conjuntamente as duas profundidades de amostragem e os quatro experimentos.

\section{RESULTADOS E DISCUSSÃO}

O solo sob mata, por não ter sido cultivado, não sofrendo os efeitos do trânsito de máquinas e equipamentos, apresentou maiores valores de porosidade total e macroporosidade, tanto na camada superficial como na de 0,10-0,20 m de profundidade, em relação ao solo sob as culturas de cobertura, em todos os experimentos (Tabelas 1 e 2), o que concorda com os resultados de Argenton et al. (2005) e Andrade et al. (2009). Apesar disso e se considerando que, de maneira geral, condições físicas do solo favoráveis ao crescimento das plantas têm sido associadas a uma porosidade de aeração mínima de 0,10 $\mathrm{m}^{3} \mathrm{~m}^{-3}$ (Dexter, 1988; Xu et al., 1992), abaixo da qual a difusão de oxigênio se torna limitante ao funcionamento das raízes, todas as coberturas propiciaram, na camada superficial, valores de macroporosidade superiores aos deste limite. Na camada de 0,10-0,20 m, os valores da macroporosidade foram, em geral, menores e, em algumas situações, inferiores ao limite de $0,10 \mathrm{~m}^{3} \mathrm{~m}^{-3}$, sinalizando algum grau de compactação.

Nas duas camadas, as diferentes coberturas do solo não diferiram em relação à porosidade total e macroporosidade nos experimentos sob preparo convencional (Tabelas 1 e 2). No experimento com feijão sob semeadura direta (Tabela 1), na camada superficial, o sorgo propiciou maior macroporosidade que o pousio enquanto na camada de 0,10-0,20 m isto se verificou com a crotalária em relação ao guandu. Foloni et al. (2006) observaram que a crotalária apresentou maior potencial de formação de "bioporos" em camadas compactadas que o guandu e, assim, melhorou as condições físicas do solo. No experimen- 
Tabela 1. Valores médios de porosidade total, macroporosidade, capacidade de aeração (CAS) e capacidade de água disponível (CAD) do solo, de acordo com as culturas de cobertura e profundidades estudadas, nos experimentos sob preparo convencional e semeadura direta com a cultura do feijão, em Santo Antônio de Goiás, G0, 2007

\begin{tabular}{|c|c|c|c|c|c|c|c|c|}
\hline \multirow{3}{*}{ Tratamento } & \multicolumn{8}{|c|}{ Profundidade (m) } \\
\hline & $0-0,10$ & $0,10-0,20$ & $0-0,10$ & $0,10-0,20$ & $0-0,10$ & $0,10-0,20$ & $0-0,10$ & $0,10-0,20$ \\
\hline & \multicolumn{2}{|c|}{ Porosidade total $\left(\mathrm{m}^{3} \mathrm{~m}^{-3}\right)$} & \multicolumn{2}{|c|}{ Macroporosidade $\left(\mathrm{m}^{3} \mathrm{~m}^{-3}\right)$} & \multicolumn{2}{|c|}{ CAS } & \multicolumn{2}{|c|}{$\mathrm{CAD}(\mathrm{mm})$} \\
\hline & \multicolumn{8}{|c|}{ Preparo convencional } \\
\hline Pousio & $0,490 a^{*}$ & $0,473 a^{*}$ & $0,118 a^{*}$ & $0,115 a^{*}$ & $0,26 a^{*}$ & $0,25 a^{*}$ & $6,7 \mathrm{a}$ & $5,3 a b^{*}$ \\
\hline Crotalária & $0,506 a^{*}$ & $0,482 a^{*}$ & $0,150 a^{*}$ & $0,112 a^{*}$ & $0,31 a^{*}$ & $0,24 a^{*}$ & $6,2 \mathrm{a}$ & $5,7 a^{*}$ \\
\hline Guandu & $0,484 a^{*}$ & $0,484 a^{*}$ & $0,110 a^{*}$ & $0,113 a^{*}$ & $0,24 a^{*}$ & $0,24 a^{*}$ & $6,1 \mathrm{a}$ & $5,5 a b^{*}$ \\
\hline Mucuna & $0,488 a^{*}$ & $0,478 a^{*}$ & $0,123 a^{*}$ & $0,111 a^{*}$ & $0,26 a^{*}$ & $0,25 a^{*}$ & $6,0 \mathrm{a}$ & $5,3 a b^{*}$ \\
\hline Sorgo & $0,498 a^{*}$ & $0,464 a^{*}$ & $0,147 a^{*}$ & $0,098 a^{*}$ & $0,30 a^{*}$ & $0,22 a^{*}$ & $5,8 \mathrm{a}$ & $4,7 \mathrm{b*}$ \\
\hline \multirow[t]{2}{*}{ C.V. $(\%)$} & 6,2 & 4,9 & 32,8 & 32,3 & 26,2 & 26,6 & 18,1 & 11,2 \\
\hline & \multicolumn{8}{|c|}{ Semeadura direta } \\
\hline Pousio & $0,482 a^{*}$ & $0,483 a^{*}$ & $0,117 b^{*}$ & $0,117 a b *$ & $0,25 b^{*}$ & $0,25 a b^{*}$ & $6,1 \mathrm{a}$ & $6,1 \mathrm{a}$ \\
\hline Crotalária & $0,502 a^{*}$ & $0,489 a^{*}$ & $0,152 a b^{*}$ & $0,133 a^{*}$ & $0,31 a b *$ & $0,28 a^{*}$ & $6,0 \mathrm{a}$ & $5,8 a^{*}$ \\
\hline Guandu & $0,508 a^{*}$ & $0,474 a^{*}$ & $0,146 a b^{*}$ & $0,090 b^{*}$ & $0,30 a b *$ & $0,20 b^{*}$ & $6,2 \mathrm{a}$ & $6,4 \mathrm{a}$ \\
\hline Mucuna & $0,497 a^{*}$ & $0,476 a^{*}$ & $0,151 a b *$ & $0,109 a b^{*}$ & $0,31 a b *$ & $0,24 a b^{*}$ & $5,9 a$ & $5,4 a^{*}$ \\
\hline Sorgo & $0,516 a^{*}$ & $0,466 a^{*}$ & $0,181 a^{*}$ & $0,102 a b^{*}$ & $0,36 a^{*}$ & $0,23 a b *$ & $5,4 a^{*}$ & $5,9 a^{*}$ \\
\hline C.V. (\%) & 5,0 & 4,1 & 27,2 & 26,0 & 22,0 & 21,8 & 14,9 & 13,4 \\
\hline Mata & 0,666 & 0,619 & 0,361 & 0,281 & 0,55 & 0,46 & 6,6 & 7,4 \\
\hline
\end{tabular}

*Médias nas colunas seguidas da mesma letra não diferem significativamente pelo teste de Tukey a $5 \%$ e as seguidas de * diferem significativamente da mata, pelo teste de Dunnett a 5\%

Tabela 2. Valores médios de porosidade total, macroporosidade, capacidade de aeração (CAS) e capacidade de água disponível (CAD) do solo, de acordo com as culturas de cobertura e profundidades estudadas, nos experimentos sob preparo convencional e semeadura direta com a cultura do milho, em Santo Antônio de Goiás, GO, 2007

\begin{tabular}{|c|c|c|c|c|c|c|c|c|}
\hline \multirow{3}{*}{ Tratamento } & \multicolumn{8}{|c|}{ Profundidade (m) } \\
\hline & $0-0,10$ & $0,10-0,20$ & $0-0,10$ & $0,10-0,20$ & $0-0,10$ & $0,10-0,20$ & $0-0,10$ & $0,10-0,20$ \\
\hline & \multicolumn{2}{|c|}{ Porosidade total $\left(\mathrm{m}^{3} \mathrm{~m}^{-3}\right)$} & \multicolumn{2}{|c|}{ Macroporosidade $\left(\mathrm{m}^{3} \mathrm{~m}^{-3}\right)$} & \multicolumn{2}{|c|}{ CAS } & \multicolumn{2}{|c|}{ CAD $(\mathrm{mm})$} \\
\hline & \multicolumn{8}{|c|}{ Preparo convencional } \\
\hline Pousio & $0,522 a^{*}$ & $0,490 a^{*}$ & $0,166 a^{*}$ & 0,117 a* & 0,33 a* & $0,24 a^{*}$ & $6,0 \mathrm{a}$ & $5,1 a^{*}$ \\
\hline Crotalária & $0,528 a^{*}$ & $0,467 a^{*}$ & $0,180 a^{*}$ & $0,084 a^{*}$ & 0,35 a* & 0,19 a* & $6,0 \mathrm{a}$ & $5,1 a^{*}$ \\
\hline Guandu & $0,524 a^{*}$ & $0,478 a^{*}$ & $0,172 a^{*}$ & $0,101 a^{*}$ & 0,34 a* & 0,22 a* & 6,1 a & 4,9 a* \\
\hline Mucuna & $0,525 a^{*}$ & $0,466 a^{*}$ & $0,170 a^{*}$ & $0,081 a^{*}$ & 0,33 a* & 0,18 a* & $6,0 \mathrm{a}$ & $5,1 a^{*}$ \\
\hline Sorgo & $0,544 a^{*}$ & $0,470 a^{*}$ & $0,198 a^{*}$ & $0,098 a^{*}$ & $0,38 a^{*}$ & $0,22 a^{*}$ & 6,1 a & $4,9 a^{*}$ \\
\hline \multirow[t]{2}{*}{ C.V. (\%) } & 5,1 & 5,5 & 23,4 & 41,9 & 18,2 & 34,9 & 13,8 & 10,4 \\
\hline & \multicolumn{8}{|c|}{ Semeadura direta } \\
\hline Pousio & $0,532 a^{*}$ & $0,494 a^{*}$ & $0,192 a^{*}$ & $0,127 a^{*}$ & $0,37 a^{*}$ & $0,27 a b^{*}$ & $5,8 \mathrm{a}$ & $5,8 a^{*}$ \\
\hline Crotalária & $0,508 a^{*}$ & 0,464 b* & $0,152 a^{*}$ & $0,091 a^{*}$ & 0,31 a* & $0,21 b^{*}$ & $5,6 a^{*}$ & $5,8 a^{*}$ \\
\hline Guandu & $0,530 a^{*}$ & $0,490 a b^{*}$ & $0,184 a^{*}$ & $0,126 a^{*}$ & 0,36 a* & $0,27 a b^{*}$ & $5,7 a$ & $5,0 a^{*}$ \\
\hline Mucuna & $0,509 a^{*}$ & $0,475 a b^{*}$ & $0,167 a^{*}$ & $0,113 a^{*}$ & 0,34 a* & $0,25 a b^{*}$ & $5,4 a^{*}$ & $5,4 a^{*}$ \\
\hline Sorgo & $0,504 a^{*}$ & $0,483 a b^{*}$ & $0,163 a^{*}$ & $0,126 a^{*}$ & 0,34 a* & $0,28 a^{*}$ & $5,7 a^{*}$ & $5,2 a^{*}$ \\
\hline C.V. (\%) & 5,7 & 4,2 & 28,2 & 22,2 & 22,4 & 17,7 & 11,3 & 10,6 \\
\hline Mata & 0,666 & 0,619 & 0,361 & 0,281 & 0,55 & 0,46 & 6,6 & 7,4 \\
\hline
\end{tabular}

*Médias nas colunas seguidas da mesma letra não diferem significativamente pelo teste de Tukey a $5 \%$ e as seguidas de * diferem significativamente da mata, pelo teste de Dunnett a $5 \%$

to com milho (Tabela 2), na camada de 0,10-0,20 m, a porosidade total do solo sob crotalária foi menor que a do solo sob pousio.

O teor de matéria orgânica do solo (M.O.) explicou mais de $60 \%$ da variação na porosidade total e na macroporosidade, tendo esses atributos aumentado com o incremento deste teor no solo, segundo as equações: $\mathrm{Pt}=$ 0,02M.O. + 0,0682, $\mathrm{R}^{2}=0.66^{* *}$ e $\mathrm{Mp}=0,0293$ M.O. $-0,489$, $\mathrm{R}^{2}=0.64^{* *}$, corroborando com os resultados obtidos por Silveira Neto et al. (2006), Juhász et al. (2007) e Andrade et al. (2009).
As coberturas também não diferiram em relação à capacidade de aeração do solo nas duas camadas dos experimentos sob preparo convencional (Tabelas 1 e 2). Com este manejo do solo, apenas a camada superficial do experimento com milho indicou valores de CAS ao redor de 0,34, considerado-o adequado, conforme Reynolds et al. (2002). Sob semeadura direta, no experimento com o feijoeiro o sorgo propiciou maior CAS que o pousio na camada superficial enquanto na camada de $0,10-0,20 \mathrm{~m}$ isto se verificou com a crotalária em relação ao guandu (Tabela 1), o que está relacionado ao efeito dessas cobertu- 
ras na macroporosidade do solo. No experimento com o milho, o sorgo propiciou maior CAS que a crotalária na camada de 0,10-0,20 m (Tabela 2). Valores de CAS iguais ou acima de 0,34 foram verificados para o sorgo na camada superficial do experimento com o feijoeiro e para todas as coberturas, com exceção da crotalária, para o experimento com o milho. A capacidade de aeração do solo também aumentou com o incremento do teor de matéria orgânica do solo, segundo a equação: CAS $=0,0477$ M.O. $-0,7368, R^{2}=0,61^{* *}$. Em todos os experimentos e nas duas camadas estudadas, o solo sob mata apresentou maiores valores de CAS que o sob as coberturas.

As coberturas diferiram significativamente quanto à capacidade de água disponível no solo (CAD) apenas na camada de 0,10-0,20 m do experimento com o feijoeiro sob preparo convencional, em que a crotalária propiciou maior valor de CAD que o sorgo. Sousa Neto et al. (2008) não encontraram diferenças entre o preparo convencional sobre pousio e a semeadura direta sobre crotalária, milheto e lab-lab (Dolichus lablab) em relação ao conteúdo de água disponível. Costa et al. (2006) e Petry et al. (2007) também não observaram diferenças na CAD entre preparo convencional do solo e semeadura direta. Em relação à mata, nos experimentos sob preparo convencional todas as coberturas propiciaram menores valores de CAD na camada de 0,10-0,20 m e não diferiram da mata na camada superficial. Sob semeadura direta, o sorgo nos experimentos com o feijoeiro e o milho, e a crotalária e a mucuna, no experimento com o milho, propiciaram menores valores de CAD na camada superficial do solo; na camada de $0,10-0,20 \mathrm{~m}$, todas as coberturas no experimen- to com o milho, e a crotalária, mucuna e sorgo no experimento com o feijoeiro, propiciaram menores valores de CAD. Borges et al. (2009) observaram que o cerrado nativo não diferiu de áreas sob pastejo quanto à CAD na camada superficial do solo. Marchão et al. (2007), por sua vez, constataram menor disponibilidade de água para as plantas na camada de $0,00-0,45 \mathrm{~m}$ de um cerrado nativo em relação a outros sistemas de manejo e uso do solo. Reynolds et al. (2002), ao compararem a CAD do solo de área de mata e sob preparo convencional ou semeadura direta, obtiveram resultados inconsistentes. Segundo esses autores, o conteúdo de água do solo no ponto de murcha (PMP) é determinado primariamente pelo seu teor de argila, que não é grandemente afetado pelo manejo do solo. A capacidade de campo (CC), por sua vez, é definida por uma interação complexa do teor de argila, densidade e matéria orgânica do solo, e mudanças nesses fatores frequentemente são compensadas, mesmo que de forma parcial, em seu impacto no valor da CC, sendo responsáveis pela inconsistência dos resultados, uma vez que a CAD depende do PMP e da CC.

A maior porosidade total, macroporosidade e CAS na camada superficial do solo sob mata em relação ao solo sob as coberturas nos experimentos sob preparo convencional, foram devidas ao maior volume de poros $\operatorname{com} \varnothing>$ $0,15 \mathrm{~mm}$ (Tabelas 3 e 4 ), uma vez que para as classes de $\varnothing$ entre 0,0002 e $0,15 \mathrm{~mm}$ a mata não diferiu das coberturas, com exceção da classe $0,15-0,075 \mathrm{~mm}$, em que a mucuna e o guandu no experimento com o feijoeiro (Tabela 3 ), e a mucuna e a crotalária no com o milho (Tabela 4), propiciaram menor volume de poros que a mata. Nesta

Tabela 3. Distribuição dos poros do solo em intervalos de tamanho, de acordo com as culturas de cobertura e profundidades estudadas, no experimento sob preparo convencional com a cultura do feijão, em Latossolo Vermelho - novembro 2007, município de Santo Antônio de Goiás, GO

\begin{tabular}{|c|c|c|c|c|c|c|c|}
\hline \multirow{3}{*}{$\begin{array}{l}\text { Diâmetro do poro } \\
(\mathrm{mm})\end{array}$} & \multicolumn{5}{|c|}{ Distribuição dos poros } & \multirow{3}{*}{ C.V. (\%) } & \multirow{3}{*}{ Mata $\left(\mathrm{m}^{3} \mathrm{~m}^{-3}\right)$} \\
\hline & Pousio & Crotalária & Guandu & Mucuna & Sorgo & & \\
\hline & \multicolumn{5}{|c|}{$\left(\mathrm{m}^{3} \mathrm{~m}^{-3}\right)$} & & \\
\hline & \multicolumn{7}{|c|}{ Camada $0,00-0,10 \mathrm{~m}$} \\
\hline$>0,15$ & $0,0848 a^{*}$ & $0,1164 a^{*}$ & $0,0796 a^{*}$ & $0,0930 a^{*}$ & $0,1151 a^{*}$ & 41,2 & 0,3226 \\
\hline $0,15-0,075$ & $0,0218 \mathrm{a}$ & $0,0221 \mathrm{a}$ & $0,0196 a^{*}$ & $0,0197 a^{*}$ & $0,0209 a$ & 17,2 & 0,0257 \\
\hline $0,075-0,05$ & $0,0114 \mathrm{a}$ & $0,0113 \mathrm{a}$ & $0,0104 \mathrm{a}$ & $0,0104 \mathrm{a}$ & $0,0106 a$ & 17,1 & 0,0126 \\
\hline $0,05-0,0375$ & $0,0074 a$ & $0,0073 \mathrm{a}$ & $0,0068 \mathrm{a}$ & $0,0068 \mathrm{a}$ & $0,0068 \mathrm{a}$ & 17,2 & 0,0079 \\
\hline $0,0375-0,03$ & $0,0054 \mathrm{a}$ & $0,0052 a$ & $0,0050 \mathrm{a}$ & $0,0049 a$ & $0,0049 a$ & 17,2 & 0,0057 \\
\hline $0,03-0,009$ & $0,0236 a$ & $0,0225 a$ & $0,0219 a$ & $0,0215 a$ & $0,0212 a$ & 17,6 & 0,0242 \\
\hline $0,009-0,0002$ & $0,0377 \mathrm{a}$ & $0,0340 a$ & $0,0342 a$ & $0,0336 a$ & $0,0321 \mathrm{a}$ & 19,8 & 0,0359 \\
\hline \multirow[t]{2}{*}{$<0,0002$} & $0,2976 a^{*}$ & $0,2877 a^{*}$ & $0,3058 a^{*}$ & $0,2987 a^{*}$ & $0,2861 a^{*}$ & 6,0 & 0,2312 \\
\hline & \multicolumn{7}{|c|}{ Camada 0,10-0,20 m } \\
\hline$>0,15$ & $0,0883 a^{*}$ & $0,0841 a^{*}$ & $0,0852 a^{*}$ & $0,0845 a^{*}$ & $0,0752 a^{*}$ & 41,5 & 0,2357 \\
\hline $0,15-0,075$ & $0,0180 a b^{*}$ & $0,0186 a^{*}$ & $0,0182 a b^{*}$ & $0,0179 a b^{*}$ & $0,0147 b^{*}$ & 15,1 & 0,0303 \\
\hline $0,075-0,05$ & $0,0092 a b^{*}$ & $0,0098 a^{*}$ & $0,0094 a b *$ & $0,0093 a b *$ & $0,0077 b^{*}$ & 13,8 & 0,0148 \\
\hline $0,05-0,0375$ & $0,0060 a b^{*}$ & $0,0064 a^{*}$ & $0,0061 a b^{*}$ & $0,0060 a b^{*}$ & $0,0050 b^{*}$ & 13,2 & 0,0094 \\
\hline $0,0375-0,03$ & $0,0043 a b^{*}$ & $0,0046 a^{*}$ & $0,0044 a b^{*}$ & $0,0043 a b *$ & $0,0037 b^{*}$ & 13,0 & 0,0066 \\
\hline $0,03-0,009$ & $0,0188 a b^{*}$ & $0,0204 a^{*}$ & $0,0194 a b^{*}$ & $0,0190 a b^{*}$ & $0,0163 b^{*}$ & 12,2 & 0,0278 \\
\hline $0,009-0,0002$ & $0,0299 a b^{*}$ & $0,0320 a^{*}$ & $0,0315 a b *$ & $0,0299 a b *$ & $0,0270 b^{*}$ & 10,9 & 0,0391 \\
\hline$<0,0002$ & $0,2986 a^{*}$ & $0,3059 a^{*}$ & $0,3103 a^{*}$ & $0,3064 a^{*}$ & $0,3143 a^{*}$ & 4,6 & 0,2550 \\
\hline
\end{tabular}

*Médias nas linhas seguidas da mesma letra não diferem significativamente pelo teste de Tukey a $5 \%$ e as seguidas de * diferem significativamente da mata, pelo teste de Dunnett a 5\%. 
Tabela 4. Distribuição dos poros do solo em intervalos de tamanho, de acordo com as culturas de cobertura e profundidades estudadas, no experimento sob preparo convencional com a cultura do milho, em Latossolo Vermelho - novembro 2007, município de Santo Antônio de Goiás, GO

\begin{tabular}{|c|c|c|c|c|c|c|c|}
\hline \multirow{3}{*}{$\begin{array}{l}\text { Diâmetro do poro } \\
\text { (mm) }\end{array}$} & \multicolumn{5}{|c|}{ Distribuição dos poros } & \multirow{3}{*}{ C.V. (\%) } & \multirow{3}{*}{ Mata $\left(\mathrm{m}^{3} \mathrm{~m}^{-3}\right)$} \\
\hline & Pousio & Crotalária & Guandu & Mucuna & Sorgo & & \\
\hline & \multicolumn{5}{|c|}{$\left(m^{3} m^{-3}\right)$} & & \\
\hline & \multicolumn{7}{|c|}{ Camada $0,00-0,10 \mathrm{~m}$} \\
\hline$>0,15$ & $0,1336 a^{*}$ & $0,1489 a^{*}$ & $0,1399 a^{*}$ & $0,1377 a^{*}$ & $0,1650 a^{*}$ & 28,0 & 0,3226 \\
\hline $0,15-0,075$ & $0,0220 a$ & $0,0207 a^{*}$ & $0,0209 a^{*}$ & $0,0218 \mathrm{a}$ & $0,0220 \mathrm{a}$ & 14,6 & 0,0257 \\
\hline $0,075-0,05$ & $0,0110 a$ & $0,0105 a$ & $0,0106 a$ & $0,0109 a$ & $0,0110 a$ & 14,2 & 0,0126 \\
\hline $0,05-0,0375$ & $0,0070 \mathrm{a}$ & $0,0068 \mathrm{a}$ & $0,0068 \mathrm{a}$ & $0,0070 \mathrm{a}$ & $0,0070 \mathrm{a}$ & 14,1 & 0,0079 \\
\hline $0,0375-0,03$ & $0,0050 \mathrm{a}$ & $0,0049 a$ & $0,0049 a$ & $0,0050 \mathrm{a}$ & $0,0051 \mathrm{a}$ & 13,8 & 0,0057 \\
\hline $0,03-0,009$ & $0,0217 a$ & $0,0213 \mathrm{a}$ & $0,0216 a$ & $0,0217 a$ & $0,0220 \mathrm{a}$ & 13,9 & 0,0242 \\
\hline $0,009-0,0002$ & $0,0329 a$ & $0,0336 a$ & $0,0345 a$ & $0,0334 a$ & $0,0344 a$ & 14,5 & 0,0359 \\
\hline \multirow[t]{2}{*}{$<0,0002$} & $0,2888 a^{*}$ & 0,2816 a* & $0,2849 a^{*}$ & $0,2878 a^{*}$ & $0,2771 a^{*}$ & 5,8 & 0,2312 \\
\hline & \multicolumn{7}{|c|}{ Camada $0,10-0,20 \mathrm{~m}$} \\
\hline$>0,15$ & $0,0929 a^{*}$ & $0,0613 a^{*}$ & 0,0771 a* & $0,0574 a^{*}$ & $0,0775 a^{*}$ & 52,4 & 0,2357 \\
\hline $0,15-0,075$ & 0,0157 a* & $0,0150 a^{*}$ & $0,0155 a^{*}$ & $0,0154 a^{*}$ & $0,0133 a^{*}$ & 16,3 & 0,0303 \\
\hline $0,075-0,05$ & 0,0083 a* & $0,0080 a^{*}$ & $0,0081 a^{*}$ & $0,0083 a^{*}$ & $0,0072 a^{*}$ & 11,7 & 0,0148 \\
\hline $0,05-0,0375$ & $0,0054 a^{*}$ & $0,0053 a^{*}$ & 0,0053 a* & $0,0054 a^{*}$ & 0,0048 a* & 10,3 & 0,0094 \\
\hline $0,0375-0,03$ & $0,0040 a^{*}$ & $0,0039 a^{*}$ & $0,0038 a^{*}$ & $0,0040 a^{*}$ & 0,0036 a* & 9,8 & 0,0066 \\
\hline $0,03-0,009$ & $0,0178 a^{*}$ & $0,0176 a^{*}$ & $0,0170 a^{*}$ & $0,0177 a^{*}$ & 0,0166 a* & 10,0 & 0,0278 \\
\hline $0,009-0,0002$ & 0,0293 a* & 0,0299 a* & $0,0278 a^{*}$ & $0,0295 a^{*}$ & 0,0290 a* & 11,5 & 0,0391 \\
\hline$<0,0002$ & 0,3160 a* & 0,3264 a* & $0,3228 a^{*}$ & $0,3284 a^{*}$ & 0,3178 a* & 5,2 & 0,2550 \\
\hline
\end{tabular}

*Médias nas linhas seguidas da mesma letra não diferem significativamente pelo teste de Tukey a $5 \%$ e as seguidas de * diferem significativamente da mata, pelo teste de Dunnett a $5 \%$

Tabela 5. Distribuição dos poros do solo em intervalos de tamanho, de acordo com as culturas de cobertura e profundidades estudadas, no experimento sob semeadura direta com a cultura do feijão, em Latossolo Vermelho - novembro 2007, município de Santo Antônio de Goiás, GO

\begin{tabular}{|c|c|c|c|c|c|c|c|}
\hline \multirow{3}{*}{$\begin{array}{l}\text { Diâmetro do poro } \\
\qquad(\mathrm{mm})\end{array}$} & & & buição dos p & & & \multirow{3}{*}{ C.V. (\%) } & \multirow{3}{*}{ Mata $\left(\mathrm{m}^{3} \mathrm{~m}^{-3}\right)$} \\
\hline & Pousio & Crotalária & Guandu & Mucuna & Sorgo & & \\
\hline & \multicolumn{5}{|c|}{$\left(m^{3} m^{-3}\right)$} & & \\
\hline & \multicolumn{7}{|c|}{ Camada 0,00 - 0,10 m } \\
\hline$>0,15$ & $0,0875 b^{*}$ & $0,1200 a b *$ & $0,1134 a b *^{*}$ & $0,1193 a b *$ & $0,1514 a^{*}$ & 35,4 & 0,3226 \\
\hline $0,15-0,075$ & $0,0193 a^{*}$ & $0,0209 a^{*}$ & $0,0215 a$ & $0,0209 a^{*}$ & $0,0201 a^{*}$ & 16,3 & 0,0257 \\
\hline $0,075-0,05$ & 0,0102 a* & $0,0106 \mathrm{a}$ & $0,0110 \mathrm{a}$ & $0,0107 a$ & $0,0100 a^{*}$ & 16,4 & 0,0126 \\
\hline $0,05-0,0375$ & $0,0068 \mathrm{a}$ & $0,0068 \mathrm{a}$ & $0,0071 \mathrm{a}$ & $0,0069 \mathrm{a}$ & $0,0064 a^{*}$ & 16,3 & 0,0079 \\
\hline $0,0375-0,03$ & $0,0049 a$ & $0,0049 a$ & $0,0052 a$ & $0,0050 \mathrm{a}$ & $0,0046 a^{*}$ & 16,2 & 0,0057 \\
\hline $0,03-0,009$ & $0,0217 a$ & $0,0214 a$ & $0,0224 a$ & $0,0214 a$ & $0,0196 a^{*}$ & 15,8 & 0,0242 \\
\hline $0,009-0,0002$ & $0,0342 \mathrm{a}$ & $0,0335 a$ & 0,0347 a & 0,0324 a & 0,0301 a & 14,7 & 0,0359 \\
\hline \multirow[t]{2}{*}{$<0,0002$} & $0,2971 a^{*}$ & $0,2835 a b *$ & $0,2922 a b^{*}$ & $0,2805 a b *$ & $0,2742 b^{*}$ & 5,5 & 0,2312 \\
\hline & \multicolumn{7}{|c|}{ Camada $0,10-0,20 \mathrm{~m}$} \\
\hline$>0,15$ & $0,0876 a b^{*}$ & 0,1021 a* & $0,0606 b^{*}$ & $0,0837 a b^{*}$ & $0,0749 a b^{*}$ & 32,7 & 0,2357 \\
\hline $0,15-0,075$ & 0,0191 a* & $0,0204 a^{*}$ & $0,0194 a^{*}$ & 0,0170 a* & $0,0177 a^{*}$ & 21,6 & 0,0303 \\
\hline $0,075-0,05$ & $0,0102 a^{*}$ & $0,0104 a^{*}$ & $0,0106 a^{*}$ & $0,0089 a^{*}$ & $0,0094 a^{*}$ & 19,0 & 0,0148 \\
\hline $0,05-0,0375$ & 0,0067 a* & 0,0067 a* & 0,0070 a* & $0,0058 a^{*}$ & 0,0062 a* & 17,6 & 0,0094 \\
\hline $0,0375-0,03$ & 0,0049 a* & 0,0048 a* & 0,0052 a* & $0,0042 a^{*}$ & 0,0045 a* & 16,9 & 0,0066 \\
\hline $0,03-0,009$ & 0,0219 a* & $0,0210 a^{*}$ & $0,0230 \mathrm{a}$ & $0,0186 a^{*}$ & 0,0204 a* & 15,4 & 0,0278 \\
\hline $0,009-0,0002$ & $0,0346 \mathrm{a}$ & 0,0324 a* & $0,0363 \mathrm{a}$ & 0,0309 a* & 0,0338 a & 12,3 & 0,0391 \\
\hline$<0,0002$ & $0,2978 a b^{*}$ & $0,2914 b^{*}$ & 0,3125 a* & $0,3063 a b *$ & $0,2990 a b^{*}$ & 4,3 & 0,2550 \\
\hline
\end{tabular}

*Médias nas linhas seguidas da mesma letra não diferem significativamente pelo teste de Tukey a $5 \%$ e as seguidas de * diferem significativamente da mata, pelo teste de Dunnett a $5 \%$

camada as coberturas não diferiram quanto à distribuição dos poros nas diversas classes de tamanho. Oliveira et al. (2002) constataram maior volume de poros com $\varnothing>$ $0,145 \mathrm{~mm}$ no solo sob Cerrado nativo em relação ao preparado com arado de discos.
Na camada de 0,10-0,20 m, no experimento com o feijoeiro, o sorgo propiciou menor volume de poros que a crotalária nas classes compreendidas entre 0,15 e $0,0002 \mathrm{~mm}$ (Tabela 3). Como o volume de poros com $\varnothing$ entre 0,0375 e $0,0002 \mathrm{~mm}$ é responsável pela retenção de água, isto ex- 
Tabela 6. Distribuição dos poros do solo em intervalos de tamanho, de acordo com as culturas de cobertura e profundidades estudadas, no experimento sob semeadura direta com a cultura do milho, em Latossolo Vermelho - novembro 2007, município de Santo Antônio de Goiás, GO

\begin{tabular}{|c|c|c|c|c|c|c|c|}
\hline \multirow{3}{*}{$\begin{array}{l}\text { Diâmetro do poro } \\
\text { (mm) }\end{array}$} & \multicolumn{5}{|c|}{ Distribuição dos poros } & \multirow{3}{*}{ C.V. (\%) } & \multirow{3}{*}{ Mata $\left(\mathrm{m}^{3} \mathrm{~m}^{-3}\right)$} \\
\hline & Pousio & Crotalária & Guandu & Mucuna & Sorgo & & \\
\hline & \multicolumn{5}{|c|}{$\left(m^{3} m^{-3}\right)$} & & \\
\hline & \multicolumn{5}{|c|}{ Camada $\quad 0-0,10 \mathrm{~m}$} & & \\
\hline$>0,15$ & 0,1609 a* & $0,1244 a^{*}$ & $0,1535 a^{*}$ & $0,1395 a^{*}$ & $0,1348 a^{*}$ & 33,3 & 0,3226 \\
\hline $0,15-0,075$ & $0,0205 a^{*}$ & $0,0185 a^{*}$ & $0,0202 a^{*}$ & $0,0182 a^{*}$ & $0,0186 a^{*}$ & 11,9 & 0,0257 \\
\hline $0,075-0,05$ & 0,0103 a* & $0,0095 a^{*}$ & $0,0101 a^{*}$ & $0,0092 a^{*}$ & $0,0094 a^{*}$ & 11,5 & 0,0126 \\
\hline $0,05-0,0375$ & $0,0066 a^{*}$ & $0,0061 a^{*}$ & $0,0065 a^{*}$ & $0,0060 a^{*}$ & $0,0061 a^{*}$ & 11,3 & 0,0079 \\
\hline $0,0375-0,03$ & $0,0047 a^{*}$ & $0,0044 a^{*}$ & 0,0047 a* & $0,0043 a^{*}$ & $0,0044 a^{*}$ & 11,4 & 0,0057 \\
\hline $0,03-0,009$ & $0,0206 a^{*}$ & $0,0195 a^{*}$ & $0,0203 a^{*}$ & $0,0189 a^{*}$ & $0,0196 a^{*}$ & 11,2 & 0,0242 \\
\hline $0,009-0,0002$ & $0,0325 \mathrm{a}$ & $0,0316 \mathrm{a}$ & $0,0322 \mathrm{a}$ & $0,0307 a^{*}$ & $0,0327 a$ & 11,7 & 0,0359 \\
\hline \multirow[t]{2}{*}{$<0,0002$} & $0,2765 a^{*}$ & $0,2937 a^{*}$ & $0,2823 a^{*}$ & $0,2823 a^{*}$ & $0,2781 a^{*}$ & 9,1 & 0,2312 \\
\hline & \multicolumn{6}{|c|}{ Camada $0,10-0,20 \mathrm{~m}$} & \\
\hline$>0,15$ & $0,0992 a b *$ & $0,0648 b^{*}$ & $0,1023 a^{*}$ & $0,0860 a b *$ & $0,1008 a b^{*}$ & 27,7 & 0,2357 \\
\hline $0,15-0,075$ & $0,0184 a^{*}$ & $0,0173 a^{*}$ & $0,0160 a^{*}$ & $0,0174 a^{*}$ & 0,0167 a* & 13,8 & 0,0303 \\
\hline $0,075-0,05$ & $0,0096 a^{*}$ & $0,0094 a^{*}$ & $0,0082 a^{*}$ & $0,0090 a^{*}$ & $0,0086 a^{*}$ & 12,6 & 0,0148 \\
\hline $0,05-0,0375$ & $0,0063 a^{*}$ & $0,0062 a^{*}$ & 0,0053 a* & $0,0059 a^{*}$ & $0,0056 a^{*}$ & 12,2 & 0,0094 \\
\hline $0,0375-0,03$ & $0,0045 a^{*}$ & $0,0045 a^{*}$ & $0,0038 a^{*}$ & $0,0043 a^{*}$ & $0,0040 a^{*}$ & 12,1 & 0,0066 \\
\hline $0,03-0,009$ & 0,0201 a* & 0,0202 a* & $0,0171 a^{*}$ & 0,0189 a* & 0,0179 a* & 11,3 & 0,0278 \\
\hline $0,009-0,0002$ & $0,0332 a^{*}$ & $0,0330 a^{*}$ & $0,0290 a^{*}$ & $0,0311 a^{*}$ & $0,0298 a^{*}$ & 10,5 & 0,0391 \\
\hline$<0,0002$ & 0,3022 a* & 0,3082 a* & $0,3082 a^{*}$ & 0,3025 a* & $0,2998 a^{*}$ & 3,6 & 0,2550 \\
\hline
\end{tabular}

*Médias nas linhas seguidas da mesma letra não diferem significativamente pelo teste de Tukey a $5 \%$ e as seguidas de $*$ diferem significativamente da mata, pelo teste de Dunnett a $5 \%$
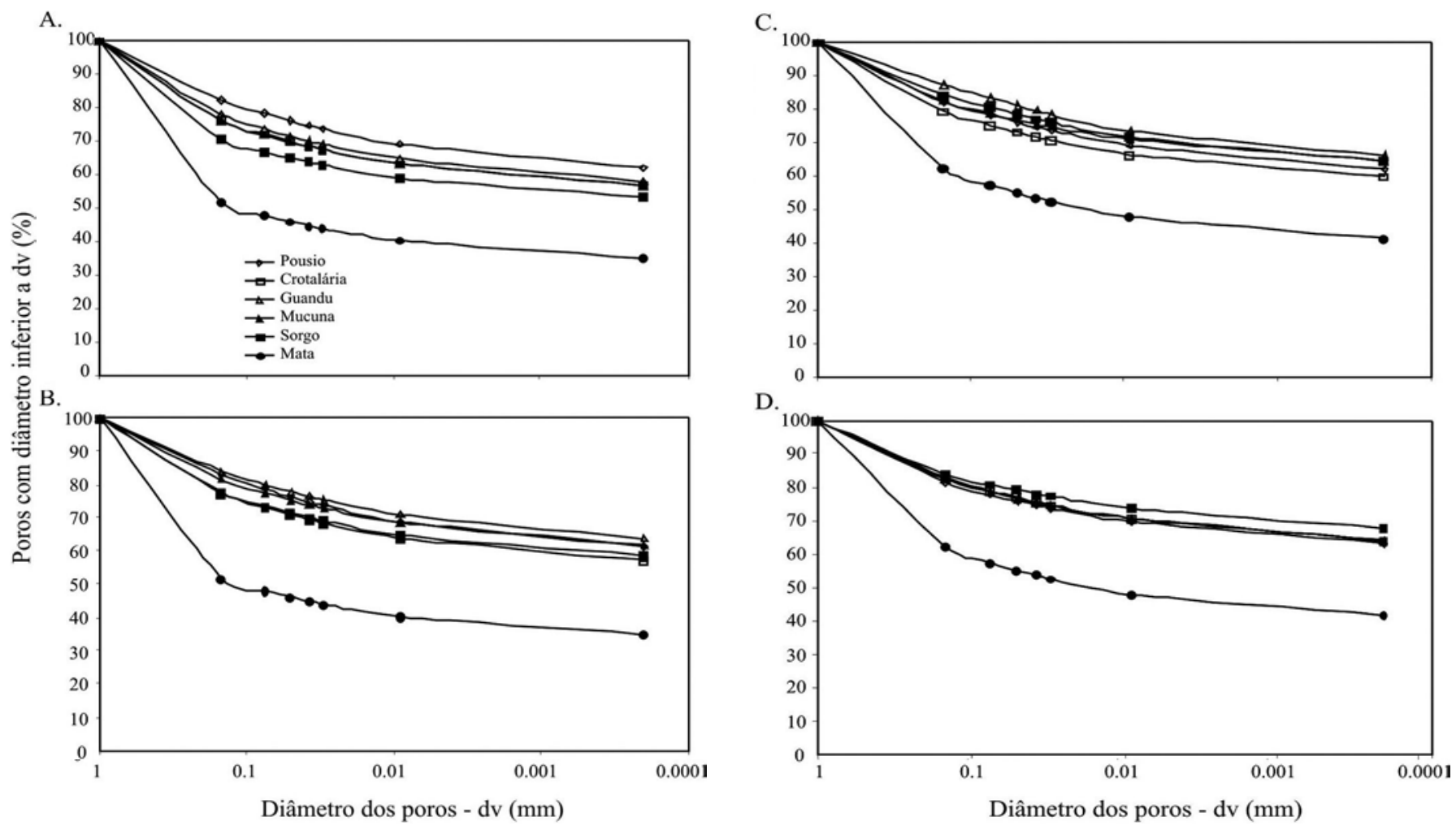

Figura 1. Curvas de distribuição de poros do solo cultivado com feijão, de acordo com as culturas de cobertura, em comparação com a mata (controle). A - preparo convencional, camada 0-10 cm; B - plantio direto, camada 0-10 cm; C - preparo convencional, camada 10-20 cm e D - plantio direto, camada 10-20 cm

plica a menor CAD do solo sob sorgo em relação ao sob crotalária (Tabela 1). No experimento com o milho as coberturas não divergiram quanto ao volume de poros (Tabela 4). Nesta camada e em todas as classes $\geq 0,0002 \mathrm{~mm}$, o solo sob mata apresentou maior volume de poros que o sob as coberturas, tanto no experimento com o feijoeiro como no com o milho, explicando sua maior porosidade total, macroporosidade, CAS e CAD; já na classe de $\varnothing<0,0002 \mathrm{~mm}$, todas as 
coberturas propiciaram maior volume de poros que a mata, nas duas camadas estudadas e nos dois experimentos (Tabelas 3 e 4), concordando com os resultados de Oliveira et al. (2002).

Sob semeadura direta, no experimento com o feijoeiro as coberturas diferiram quanto ao volume de poros apenas nas classes $>0,15 \mathrm{~mm}$ e $<0,0002 \mathrm{~mm}$. Na camada superficial o sorgo propiciou maior volume de poros na classe $>0,15 \mathrm{~mm}$ em relação ao pousio enquanto na camada de 0,10-0,20 $\mathrm{m}$ isto se verificou com a crotalária em relação ao guandu (Tabela 5), explicando a maior macroporosidade e CAS do sorgo e da crotalária, respectivamente, em relação ao pousio e ao guandu (Tabela 1). O maior volume de poros com grande $\varnothing(>0,15 \mathrm{~mm})$ foi contrabalançado pela diminuição no volume de poros na classe < $0,0002 \mathrm{~mm}$, em que sorgo e crotalária apresentaram menores valores que o pousio e o guandu, respectivamente.

No experimento com o milho as coberturas diferiram apenas na camada de 0,10-0,20 m, em que o guandu propiciou maior volume de poros com $\varnothing>0,15 \mathrm{~mm}$ em relação à crotalária (Tabela 6). Nesta camada, referida leguminosa mostrou menor porosidade total e CAS que outras coberturas (Tabela 2). Como as coberturas não diferiram com relação ao volume de poros responsáveis pela retenção da água no solo, $0,0002 \leq \varnothing \leq 0,0375 \mathrm{~mm}$ (Tabelas 5 e 6), também não divergiram em relação a CAD (Tabelas 1 e 2). Os melhores atributos físicos do solo sob mata na camada superficial foram devidos, basicamente, ao maior volume de poros com $\varnothing>0,075 \mathrm{~mm}$, no experimento com o feijoeiro (Tabela 5), e aos com $\varnothing>0,009 \mathrm{~mm}$, no experimento com o milho (Tabela 6). Oliveira et al. (2002) observaram que a camada superficial do solo sob semeadura direta apresentou menor volume de poros com $\varnothing>0,145 \mathrm{~mm}$ que a do sob Cerrado nativo, explicando sua menor macroporosidade.

$\mathrm{Na}$ camada de $0,10-0,20 \mathrm{~m}$ e em todas as classes $\geq 0,0002$ $\mathrm{mm}$, o solo sob mata indicou maior volume de poros que o sob as coberturas, ocorrendo o contrário na classe < $0,0002 \mathrm{~mm}$, nas duas camadas estudadas, tanto no experimento com o feijoeiro como no com o milho (Tabelas 5 e 6). A menor CAD nas duas camadas do solo sob sorgo em relação ao sob mata, no experimento com o feijoeiro (Tabela 1), foi devida ao menor volume de poros com $\varnothing$ entre 0,009 e $0,0375 \mathrm{~mm}$. Com relação à crotalária e à mucuna, a menor CAD na camada de $0,10-0,20 \mathrm{~m}$ se deveu ao menor volume de poros com $\varnothing$ entre $0,0002 \mathrm{e}$ $0,0375 \mathrm{~mm}$ (Tabela 5). No experimento com o milho a menor CAD na camada superficial do solo sob crotalária, sorgo e mucuna em relação ao sob mata (Tabela 2) foi devida ao menor volume de poros com $\varnothing$ entre 0,009 e $0,0375 \mathrm{~mm}$, no caso das duas primeiras coberturas, e ao menor volume de poros com $\varnothing$ entre 0,0002 e $0,0375 \mathrm{~mm}$, no caso da mucuna (Tabela 6). Esses tamanhos de poros foram responsáveis pela maior CAD na camada de 0,10 $0,20 \mathrm{~m}$ do solo sob mata em relação ao sob as coberturas. Spera et al. (2000) constataram que o teor de água disponível em um Latossolo Vermelho estava relacionado ao volume de poros entre 0,003 e $0,030 \mathrm{~mm}$ de diâmetro.

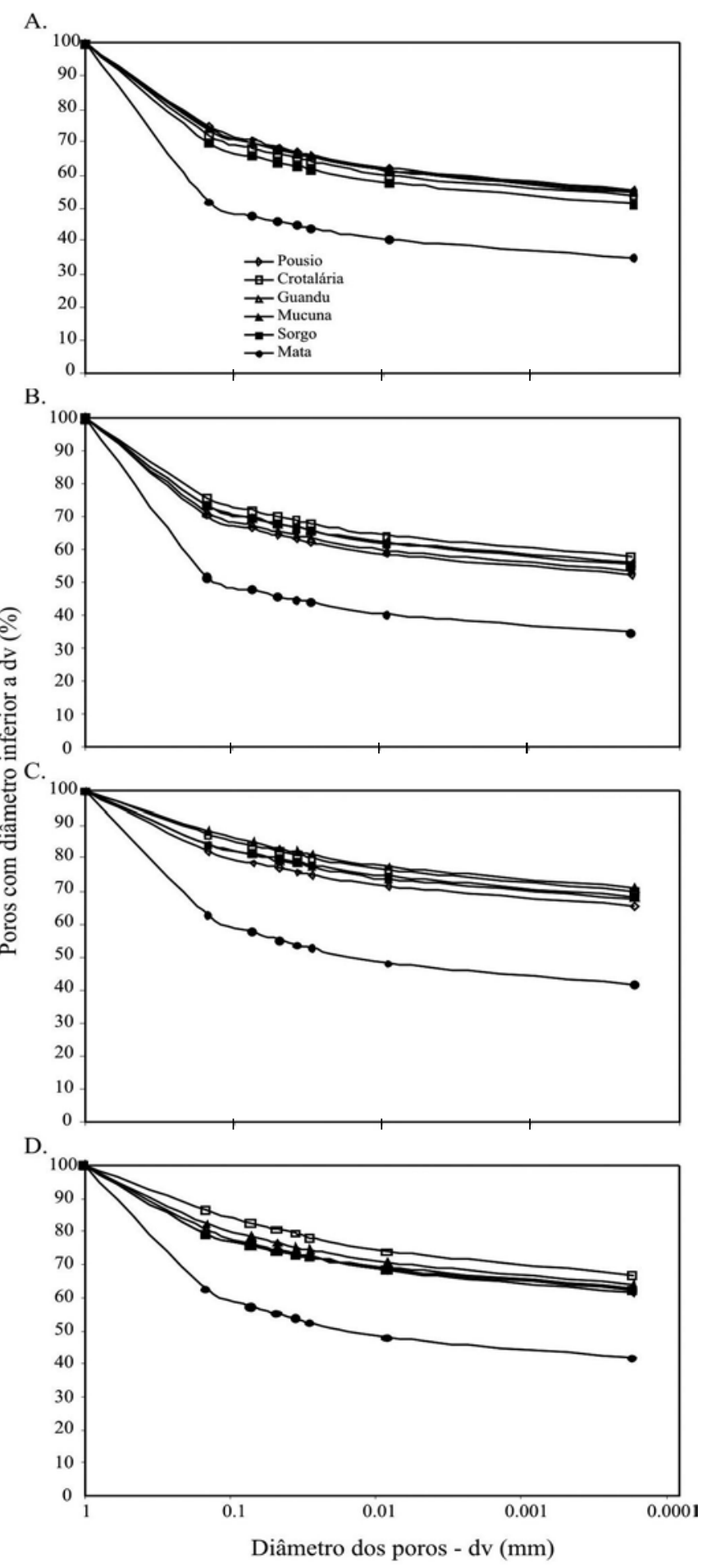

Figura 2. Curvas de distribuição de po ros do solo cultivado com milho, de acordo com as culturas de cobertura, em comparação com a mata (controle). A - preparo convencional, camada $0-10 \mathrm{~cm}$; B - plantio direto, camada 0-10 cm; C - preparo convencional, camada $10-20 \mathrm{~cm}$ eD - plantio direto, camada $10-20 \mathrm{~cm}$

Analisando os quatros experimentos observa-se que a maior proporção dos poros do solo sob as coberturas, especialmente na camada de $0,10-0,20 \mathrm{~m}$, se encontra na classe de $\varnothing<0,0002 \mathrm{~mm}$, que são aqueles poros nos quais a água pode permanecer retida com energia muito alta sendo, portanto, indisponível às plantas, o que justifica os valores relativamente baixos de CAD e sinaliza que o solo 
pode apresentar problemas com relação à infiltração e disponibilidade de água para as plantas.

Por meio da curva de distribuição de poros do solo pode-se comparar graficamente os tratamentos. De maneira geral, quanto maior for a inclinação dessa curva maior tende a ser a diversidade do tamanho dos poros. Desta forma, observa-se que o solo sob mata foi o que apresentou maior diversidade de tamanho de poros (Figuras 1 e 2), indicando maior capacidade de agregação (Ribeiro et al., 2007). Pela análise da Figura 1 verifica-se a maior diversidade de tamanho de poros na camada superficial do solo sob sorgo em relação ao sob pousio (Figura 1B) e da crotalária em relação ao guandu (Figura 1D) na camada de $0,10-0,20 \mathrm{~m}$, no experimento com o feijoeiro sob semeadura direta. Nesta camada, no experimento com o milho, ao contrário, o solo sob crotalária indicou menor diversidade de tamanho de poros (Figura 2D). Esses resultados corroboram com o discutido anteriormente.

\section{CONCLUSÕES}

1. O uso do solo sob vegetação de cerrado para a produção agrícola resultou, independentemente do sistema de cultivo, em modificações nos seus atributos físicos, reduzindo a macroporosidade, a porosidade total e a capacidade de aeração do solo.

2. Os atributos físicos do solo foram afetados favoravelmente por seu conteúdo de matéria orgânica.

3. As variações entre as coberturas e entre essas e a mata na porosidade total, macroporosidade, capacidade de aeração e capacidade de água disponível no solo, podem ser explicadas pela variação na distribuição do tamanho de poros do solo.

4. A melhor qualidade física da camada superficial do solo sob mata em relação à sob as coberturas no preparo convencional foi devida, basicamente, ao volume de poros com $\varnothing>0,075 \mathrm{~mm}$. Sob semeadura direta, o mesmo se verificou no cultivo do feijoeiro, enquanto no do milho se deveu ao volume de poros com $\varnothing>0,009 \mathrm{~mm}$.

\section{LITERATURA CITADA}

Andrade, R. da S.; Stone, L. F.; Silveira, P. M. da. Culturas de cobertura e qualidade física de um Latossolo em plantio direto. Revista Brasileira de Engenharia Agrícola e Ambiental, v.13, p.411-418, 2009.

Argenton, J.; Albuquerque, J. A.; Bayer, C.; Wildner, L. do P. Comportamento de atributos relacionados com a forma da estrutura de Latossolo Vermelho sob sistemas de preparo e plantas de cobertura. Revista Brasileira de Ciência do Solo, v.29, p.425-435, 2005.

Borges, T. A.; Oliveira, F. A.; Silva, E. M. da; Goedert, W. J. Avaliação de parâmetros físico-hídricos de Latossolo Vermelho sob pastejo e sob cerrado. Revista Brasileira de Engenharia Agrícola e Ambiental, v.13, p.18-25, 2009.
Costa, E. A.; Goedert, W. J.; Sousa, D. M. G. de. Qualidade do solo submetido a sistemas de cultivo com preparo convencional e plantio direto. Pesquisa Agropecuária Brasileira, v.41, p.1185-1191, 2006.

Dexter, A. R. Advances in characterization of soil structure. Soil \& Tillage Research, v.11, p.199-238, 1988.

EMBRAPA - Empresa Brasileira de Pesquisa Agropecuária. Centro Nacional de Pesquisa de Solos. Manual de métodos de análise de solos. 2.ed. Rio de Janeiro: Embrapa CNPS, 1997. 212p. Documentos, 1.

Foloni, J. S. S.; Lima, S. L. de; Büll, L.T. Crescimento aéreo e radicular da soja e de plantas de cobertura em camadas compactadas de solo. Revista Brasileira de Ciência do Solo, v.30, p.49-57, 2006.

Freitas Júnior, E.; Silva, E. M. Uso da centrífuga para a determinação da curva de retenção de água no solo, em uma única operação. Pesquisa Agropecuária Brasileira, v.19, p.1423-1428, 1984.

Genuchten, M. T. van. A closed form equation for predicting the hydraulic properties of unsaturated soils. Soil Science Society of America Journal, v.44, p.892-898, 1980.

Juhász, C. E. P.; Cooper, M.; Cursi, P. R.; Ketzer, A. O.; Toma, R. S. Savanna woodland soil micromorphology related to water retention. Scientia Agrícola, v.64, p.344-354, 2007.

Kay, B. D.; Vandenbygaart, A. J. Conservation tillage and depth stratification of porosity and soil organic matter. Soil \& Tillage Research, v.66, p.107-118, 2002.

Marchão, R. L.; Balbino, L. C.; Silva, E. M. da; Santos Junior, J. de D. G.; Sá, M. A. C. de; Vilela, L.; Becquer, T. Qualidade física de um Latossolo Vermelho sob sistemas de integração lavoura-pecuária no Cerrado. Pesquisa Agropecuária Brasileira, v.42, p.873-882, 2007.

Oliveira, G. C.; Dias Júnior, M. S.; Resck, D. V. S.; Curi, N.; Moraes, M. F. Distribuição de poros e retenção de água em um Latossolo Vermelho argiloso sob experimentos de uso e manejo de longa duração. In: Reunião Brasileira de Manejo e Conservação do Solo e da Água, 14, 2002, Cuiabá. Anais...Viçosa: Sociedade Brasileira de Ciência do Solo, 2002. CD-Rom.

Petry, M. T.; Zimmermann, F. L.; Carlesso, R.; Michelon, C. J.; Kunz, J. H. Disponibilidade de água do solo ao milho cultivado sob sistemas de semeadura direta e preparo convencional. Revista Brasileira de Ciência do Solo, v.31, p.531-539, 2007.

Reynolds, W. D.; Bowman, B. T.; Drury, C. F.; Tan, C. S.; Lu, X. Indicators of good soil physical quality: Density and storage parameters. Geoderma, v.110, p.131-146, 2002.

Ribeiro, K. D.; Menezes, S. M.; Mesquita, M. da G. B. de F.; Sampaio, F. de M. T. Propriedades físicas do solo, influenciadas pela distribuição de poros, de seis classes de solos da região de Lavras, MG. Ciência e Agrotecnologia, v.31, p.1167-1175, 2007.

SAS Institute Inc. (Cary, NC). SAS/STAT procedure guide for personal computers: version 5. Cary, NC, 1999.

Silva, S. C.; Xavier, L. de S.; Santana, N. M. P. de; Cardoso, G. M.; Pelegrini, J. C. Informações meteorológicas para pesquisa e planejamento agrícola referentes ao município de Santo Antônio de Goiás, GO, 2001. Santo Antônio de Goiás: Embrapa Arroz e Feijão, 2002. 21p. Documentos, 136.

Silveira Neto, A. N. de; Silveira, P. M. da; Stone, L. F.; Oliveira, L. F. C. de. Efeitos de manejo e rotação de culturas em atributos físicos do solo. Pesquisa Agropecuária Tropical, v.36, p.29-35, 2006. 
Sousa Neto, E. L. de; Andrioli, I.; Beutler, A. N.; Centurion, J. F. Atributos físicos do solo e produtividade de milho em resposta a culturas de pré-safra. Pesquisa Agropecuária Brasileira, v.43, p.255-260, 2008.

Spera, S. T.; Reatto, A.; Correia, J. R.; Silva, J. C. S. Características físicas de um Latossolo Vermelho-Escuro no Cerrado de Planaltina, DF, submetido à ação do fogo. Pesquisa Agropecuária Brasileira, v.35, p.1817-1824, 2000.
Stone, L. F.; Silveira, P. M. da. Efeito do sistema de preparo na compactação do solo, disponibilidade hídrica e comportamento do feijoeiro. Pesquisa Agropecuária Brasileira, v.24, p.8391, 1999.

Xu, X.; Nieber, J. L.; Gupta, S. C. Compaction effect on the gas diffusion coefficient in soils. Soil Science Society of America Journal, v.56, p.1743-1750, 1992. 